Mitteilungen der Österreichischen Geographischen Gesellschaft, 160. Jg., S. 387-391

(Annals of the Austrian Geographical Society, Vol. 160, pp. 387-391)

Wien (Vienna) 2018, https://doi.org/10.1553/moegg160s387

\title{
NUN SIND ES IN WIEN DOCH EIN PAAR JAHRE MEHR GEWORDEN ... Hans-Heinrich Blotevogel Zu SEINEM 75. Geburtstag
}

\author{
Elisabeth GRUBER, Wien*
}

mit 2 Abb. im Text

Es ist bereits sechs Jahre her, dass Hans-Heinrich BLotevogel die Vertretungsprofessur für „Angewandte Geographie, Raumforschung und Raumordnung“ am Institut für Geographie und Regionalforschung der Universität Wien angetreten hat. Nachdem Heinz FASSMANN zum Vizerektor der Universität Wien ernannt worden war, war es wichtig, einen vorübergehenden Ersatz mit großer Lehrerfahrung zu finden. Schnell stellte sich heraus, dass der neue Vertretungsprofessor die exakt richtige Wahl für die Arbeitsgruppe war, um im Bereich der Raumforschung und Raumordnung am Wiener Geographie-Institut qualitätsvoll zu lehren. Es zeigte sich überdies, dass er auch bei der Erfüllung von Aufgaben jenseits der Lehre zu einem wichtigen Teil sowohl der Arbeitsgruppe als auch des Instituts wurde, und nachdem sich die Karenz von Heinz FASSMANN nachhaltig verlängerte, wurde aus einer kurzfristigen Vertretung eine längerfristige Aufgabe für BLOTEVoGELs dritten Lebensabschnitt.

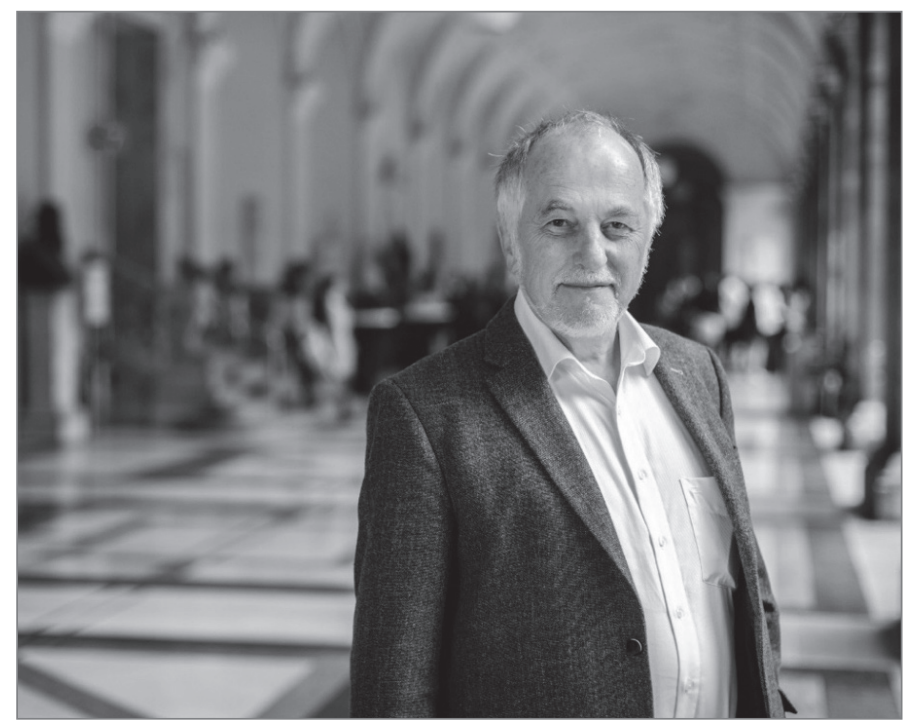

Hans-Heinrich Blotevogel (Foto: (C) D. Dutkowski/Universität Wien)

\footnotetext{
* Mag. Dr. Elisabeth Gruber, Universität Wien, Institut für Geographie und Regionalforschung, Universitätsstraße 7, A-1010 Wien; E-Mail: elisabeth.gruber@univie.ac.at
} 


\section{Auf Exkursion mit einer Koryphäe}

Als Universitätsassistentin lernte ich Hans BLotevogel im Rahmen einer seiner ersten Lehrveranstaltungen am Wiener Institut für Geographie und Regionalforschung kennen; es handelte sich um seine erste Auslandsexkursion mit Wiener Studierenden, die ich auch begleiten sollte. Neben Vorfreude fühlte ich damals große Ehrfurcht, kannte man ihn doch vor allem für seine Beiträge zur Wissenschaftstheorie in der Geographie und als einen der anerkanntesten Professoren in der deutschsprachigen Geographie und Raumordnung. Eine Recherche zu seiner Person ergab, mit welch` renommierter Persönlichkeit ich 14 Tage im Ruhrgebiet verbringen sollte. Bis zum damaligen Zeitpunkt hatte Blotevogel mehr als 190 wissenschaftliche Arbeiten publiziert und wurde über 2.500 Mal zitiert. Mit einem „Hirsch-Faktor“ (h-Index) von 30 zählte er zu den meist zitierten deutschsprachigen Humangeographen. Neben seinen zahlreichen Publikationen wies er auch einen sehr großen Bekanntheitsgrad in der Fachwelt auf - auch über die Grenzen des deutschen Sprachraums hinaus. Im Rahmen seiner Professuren an der Gerhard-Mercator-Universität Duisburg und später an der Technischen Universität Dortmund betreute er zahlreiche, heute namhafte und wissenschaftlich erfolgreiche Absolventinnen und Absolventen. Seine Bedeutung in der Geographie bestätigt sich durch immer noch aufrechte Netzwerke, Einladungen zu Diskussionen und Vorträgen und eine anhaltende Zunahme der Zitationen seiner Werke. In Deutschland prägte er den Diskurs und die Definition der Metropolregionen sowie die Fortentwicklung des Zentrale-Orte-Konzepts. Im Bereich der theoretischen Geographie lieferte er wesentliche Beiträge in Bezug auf Überlegungen zu Raum und Region und trug auch zu einer Weiterentwicklung der Paradigmendiskussion in der Humangeographie bei. Neben Raumordnung und Regionalentwicklung publizierte BLOTEVOGEL unter anderem auch zu den Themen Stadtentwicklung und räumliche Mobilität.

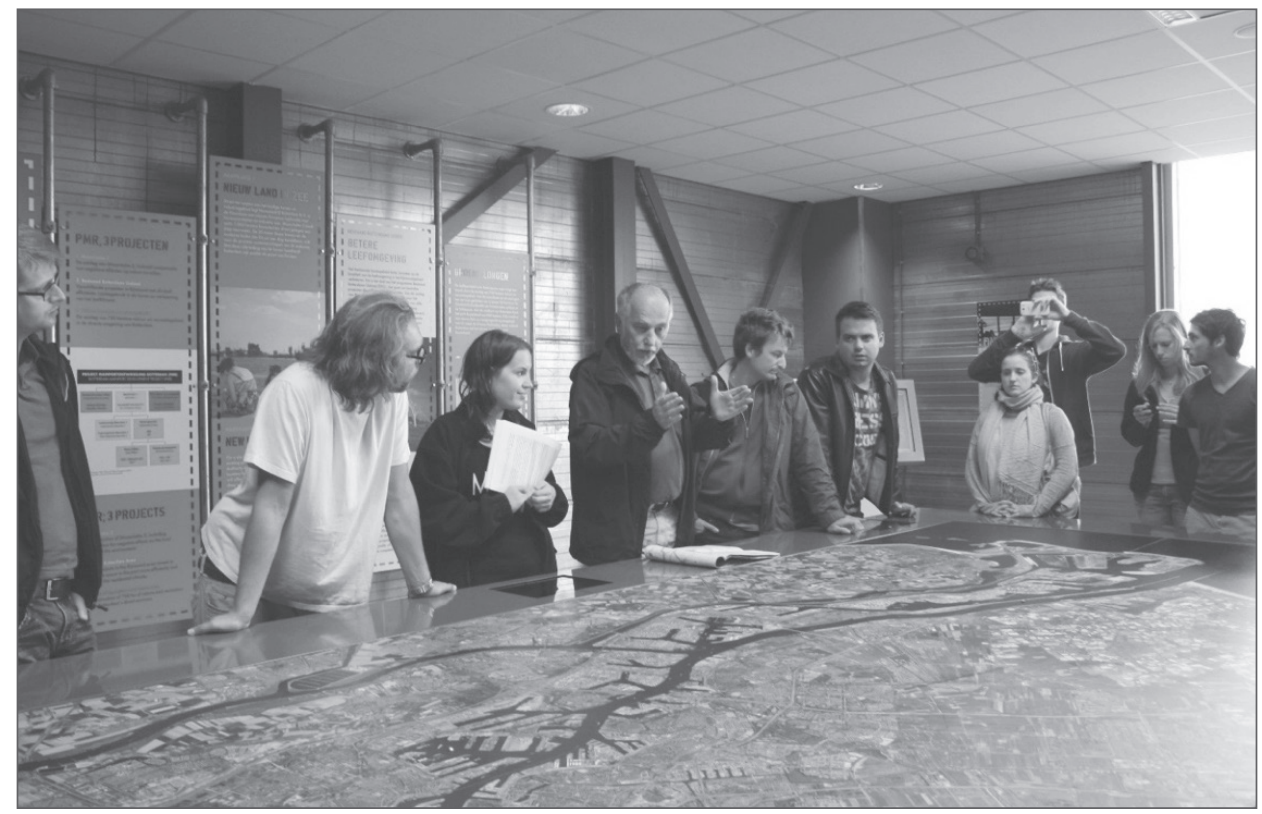

Auf Exkursion in den Niederlanden, am Hafengelände in Rotterdam (Foto: (C) Elisabeth GRUBER) 
Mit diesem Wissen und mit großem Respekt fuhr ich auf Exkursion nach Nordwestdeutschland und in die Niederlande, um mit ihm gemeinsam die Studierenden zu betreuen. Umso überraschter stellte ich fest, dass der „Herr Professor“ mehr war als ein großer und erfahrener Wissenschaftler. Neben spannenden Inputs während der Exkursion ließ es sich mit ihm - abends nach dem Exkursionsprogramm - auch über nicht-wissenschaftliche Themen diskutieren. Es stellte sich heraus, dass er nicht nur einen beeindruckenden fachlichen Lebenslauf hat, sondern auch eine unterhaltsame und freundliche Privatperson ist. In den letzten Jahren - unter anderem auf zwei weiteren Exkursionen, als Zweitbetreuer meiner Dissertation, im Rahmen von Lehrveranstaltungen und bei diversen Heurigenbesuchen - hatte ich die Möglichkeit, ihn fachlich und privat kennen und schätzen zu lernen und neben seinem großen Wissen auch von seinen Kompetenzen und „Softskills“ zu profitieren. Denn Hans Blotevogel ist nicht nur ein guter Professor, sondern auch ein guter Chef und sympathischer Kollege.

Mit der Würdigung seiner Person zum 75. Geburtstag möchte ich mich, stellvertretend für unsere Arbeitsgruppe für Angewandte Geographie, Raumforschung und Raumordnung ${ }^{1)}$ und das Institut für Geographie und Regionalforschung, bedanken: für die unverzichtbaren Beiträge zur Lehre, aber auch zur Administration und Leitung unserer Arbeitsgruppe, sowie für die wichtigen Einflüsse auf die Entwicklung des Instituts. Die Würdigung soll nicht nur Hans Blotevogel ehren, sondern auch zeigen, dass er in vielen Bereichen ein bemerkenswertes Vorbild für andere Wissenschaftlerinnen und Wissenschaftler ist.

\section{Lehre mit Leidenschaft}

Es strömt wie aus „Schaffeln“, der Exkursionstag ist eigentlich schon seit einer halben Stunde vorbei, dennoch lässt es sich der Herr Professor nicht nehmen, noch eine weitere wichtige Ausführung anzubringen. Die Versuche der Assistentin, ihn dabei zu stoppen, sind vergeblich. Denn: „Eines muss ich noch sagen ...". Hans Blotevogel hat sich in seiner Laufbahn ein unglaublich großes Wissen angeeignet. Neben Geographie studierte er auch Soziologie, Philosophie und (wie viele der Studierenden wissen, die von ihm bei Abschlussarbeiten betreut wurden) Germanistik in Münster und Tübingen. An der Ruhr-Universität Bochum promovierte er 1972 und habilitierte sich anschließend 1980 im Fach Geographie; seine erste Professur, die er bis 2004 innehatte, trat er im Jahr 1983 an der Universität Duisburg an. Anschließend wechselte er an die Technische Universität Dortmund, wo er von 2005 bis 2008 Geschäftsführender Direktor des Instituts für Raumplanung (IRPUD) war und bis zum Erreichen der Altersgrenze 2009 blieb.

Sein umfassendes Studium, aber auch die Aktivitäten in diversen Gremien an außeruniversitären Instituten und Einrichtungen (u. a. Akademie für Raumforschung und Landesplanung, ARL; Deutsche Gesellschaft für Geographie, DGfG) haben dazu geführt, dass er viele Prozesse und Entwicklungsschritte in der Raumordnung und Regionalentwicklung selbst miterlebt hat, was ihm die Fähigkeit gibt, diese nicht trocken und anhand von Lehrbuchbeispielen aufzuzählen, sondern sie den Studierenden, aber auch den Kolleginnen und Kollegen mit viel Detail- und Insiderwissen anhand konkreter Beispiele näherzubringen. So wissen vermutlich alle Absolventinnen und Absolventen des Masterstudiums Raumforschung und Raumordnung, die in den letzten Jahren ihr Studium am Wiener Institut abgeschlossen haben, wie sich am Beispiel der Internationalen Bauausstellung (IBA) Emscher-Park die Entwicklung des perspektivischen Inkrementalismus in der deutschen Raumordnung beschreiben lässt und sind auch informiert, dass die Entstehung von „Business Improvement

\footnotetext{
1) Bei der Erstellung des Textes waren Angelika Horvath, Alois Humer, Petra KöcK und Jiannis Kaucic unverzichtbar: Danke für eure Unterstützung!
} 
Districts“ im deutschsprachigen Raum im Bochumer „Bermudadreieck“ ihren Ausgang genommen hat.

In Wien unterrichtete Blotevogel bis zum heutigen Datum in über 100 Lehrveranstaltungen (Vorlesungen, Proseminare, Seminare, Exkursionen, Praktika, Privatissima). Zusätzlich betreute er seit 2012 am Wiener Institut für Geographie und Regionalforschung 31 Masterarbeiten, 10 Magister- und Diplomarbeiten und mehr als 100 Bachelorarbeiten. Bei Dissertationsprojekten übernahm er oft die Rolle des Zweitbetreuers, und er wirkte in unzähligen Defensiones von Masterarbeiten und bei zahlreichen Diplomprüfungen als Prüfer mit. Dass er seine Tätigkeit in Wien schlussendlich mehr als einmal verlängert hat, ergab sich aufgrund seiner großen Leidenschaft, zu lehren und Wissen an die Studierenden weiterzugeben.

\section{Detailwissen und dennoch Gesamtgeograph}

„Aber dazu kann ich eigentlich gar nicht so viel sagen ...", lautete oft der Einleitungssatz von Hans Blotevogel, bevor er einen seiner umfassenden Vorträge, etwa über die Bedeutung von Gewerbeparks als Motoren der wirtschaftlichen Entwicklung, über Architekturepochen und bauliche Ensembles (z. B. Torsituationen) sowie spezielle Architekturstile in Nordeuropa (Backsteinexpressionismus) hielt und nebenbei über Eiszeitschwellen in Mitteldeutschland referierte.

Aufgrund der thematischen Vielfalt seiner Forschungsinteressen gilt Hans BLOTEVoGeL als Stadt-, Wirtschafts- und Sozialgeograph. Er ist jedoch auch mit Inhalten aus verwandten Disziplinen vertraut und besitzt einen ungemeinen Schatz an Allgemeinwissen. Auch in den Grundlagen der Physischen Geographie hat er fundierte Kenntnisse. Während seiner Zeit an der Universität Duisburg leitete er deshalb auch Exkursionen in den Alpenraum und referierte vor Ort über den geologisch-tektonischen Aufbau der Alpen.

Exkursionen kamen daher, auch wenn Sie grundsätzlich im Themenbereich der Raumordnung angesiedelt waren, nie ohne Inhalte der Landschaftsgenese oder grundlegende historisch-gesellschaftliche Grundlagen der Kulturlandschaft aus. Einen Widerspruch stellt das für Blotevogel, ganz im Sinne von Alexander von HumBoldt, nicht dar, denn er vertritt die Meinung, dass eine umfassende Information über Raum und Gesellschaft notwendig ist, um eben diese zu erforschen und zu „ordnen“. Dennoch ist sein enormer Wissensschatz keine Selbstverständlichkeit. Gesamtgeographisches Wissen können heute nur mehr die Wenigsten vorweisen. BLOTEVoGEL motivierte aber stets dazu, auch über den Tellerrand zu blicken. Gelebte integrative Geographie und Interdisziplinarität als Asset konnte er als Paradebeispiel auf diese Weise allen seinen Studierenden und auch Kolleginnen und Kollegen am Institut gut vermitteln.

Überdies zeigte sich BLOTEVOGEL trotz fortgeschrittener Karriere stets an neuen Inhalten des Faches interessiert. Keine Frage, er gibt es offen zu, dass es ihm Mühe bereitet, mit allen Entwicklungen der Forschung Schritt zu halten. Er weiß allerdings ob der Notwendigkeit, aktuell informiert zu sein. Mit seinem Wechsel nach Wien begann er auch, sich in die österreichische Medienlandschaft einzulesen. „Lesen Sie Zeitung, um informiert zu sein“, predigt er unseren Studierenden bis heute.

\section{Mehr als nur eine Vertretung: Kollegialer Chef mit Erfahrung}

Hans Blotevogel war aber nicht nur ein Vertretungsprofessor, der sich um die Betreuung der Studierenden kümmerte. Er engagierte sich mit bewundernswertem Einsatz sowohl für die Anliegen der Arbeitsgruppe als auch für jene des Instituts. Im Jahr 2012, als er an das Wiener Institut kam, waren zwei Professuren vakant, bei deren Besetzung er in einer Kommission mitwirkte. Er über- 
nahm zudem die Organisation einer Habilitationskommission, wirkte in der Curricularkommission (Reform des Bachelorstudiums und der Masterstudien im Fach Geographie) mit, war Mitglied der Studienkonferenz und im Jahr 2015 wurde er sogar noch stellvertretender Institutsvorstand.

Der Grund für seine Aktivität in so vielen Beiräten war bestimmt auch der Tatsache geschuldet, dass dem neuen Professor schon kurz nach seiner Ankunft großes Vertrauen entgegengebracht wurde. Einerseits hatte er viel Erfahrung und Wissen im Umgang mit diversen Akteuren gesammelt, war er doch bereits zuvor als Professor, Vizedekan und Prorektor an der Universität Duisburg aktiv und kannte die Abläufe und die Funktionsweise von Universitätsgremien. Auch wenn die Universität Wien eigenen Abläufen folgt, so lernten wir, dass das System „Universität“ überall sehr ähnlich funktioniert: ob im Ruhrpott oder in Ostösterreich. Andererseits war aber sicherlich entscheidend, dass Hans Blotevogel einen sehr beeindruckenden Führungsstil pflegt, der es ihm ermöglicht, locker und dennoch ernsthaft, erfahren und gleichzeitig nicht autoritär, sowie bestimmt und zielstrebig, und dennoch offen für Neues an Problemstellungen heranzutreten. Damit gelang es ihm, trotz seines schon fortgeschrittenen Alters und trotz oder gerade wegen seiner langjährigen universitären Berufserfahrung, ,frischen Wind“ in das Institut zu bringen.

\section{In Zukunft: Wien?}

Nach mehrmaliger Ankündigung hat sich Hans Blotevogel mittlerweile aus seiner leitenden Funktion verabschiedet und die Arbeitsgruppe freute sich, Axel PRIEBS als neuen Inhaber der Vertretungsprofessur willkommen zu heißen. Dennoch bleibt uns Blotevogel aber noch in Wien erhalten. Für die Arbeitsgruppe ist er ein unverzichtbarer Lehrbeauftragter und ein wichtiger Berater in vielen verschiedensten Bereichen - von der akademischen Selbstverwaltung im Institut und in der Fakultät über die Lehre bis zur Projektberatung.

Nicht zuletzt hat Hans Blotevogel in den vielen Jahren aber auch - wie viele von uns - eine besondere Liebe zu Wien entdeckt. Endlich gibt es nun mehr Zeit, die Stadt zu genießen. Ganz im Sinn der angewandten Geographie werden neue Wanderrouten erschlossen und bislang unbekannte Heurigenlokale entdeckt.

Die Arbeitsgruppe freut sich, auf ihn bis auf Weiteres nicht verzichten zu müssen. Im November 2018 haben wir bereits persönlich mit ihm auf seinen 75. Geburtstag angestoßen.

Nun aber auch auf diesem Wege: Alles Gute, lieber Hans! 